\title{
MAXIMAL AND LINEARLY INEXTENSIBLE POLYNOMIALS
}

\author{
JULIUS BORCEA
}

\begin{abstract}
Let $S(n, 0)$ be the set of monic complex polynomials of degree $n \geq 2$ having all their zeros in the closed unit disk and vanishing at 0 . For $p \in S(n, 0)$ denote by $|p|_{0}$ the distance from the origin to the zero set of $p^{\prime}$. We determine all 0 -maximal polynomials of degree $n$, that is, all polynomials $p \in S(n, 0)$ such that $|p|_{0} \geq|q|_{0}$ for any $q \in S(n, 0)$. Using a second order variational method we then show that although some of these polynomials are linearly inextensible, they are not locally maximal for Sendov's conjecture.
\end{abstract}

\section{Introduction}

Let $S_{n}$ be the set of all monic complex polynomials of degree $n \geq 2$ having all their zeros in the closed unit disk $\bar{D}$. If $p \in S_{n}$ and $a \in Z(p)$ then the GaussLucas theorem implies that $(a+2 \bar{D}) \cap Z\left(p^{\prime}\right) \neq \emptyset$, where $Z(p)$ and $Z\left(p^{\prime}\right)$ denote the zero sets of $p$ and $p^{\prime}$, respectively. In 1959 Sendov conjectured that this result may be substantially improved in the following way:

Conjecture 1.1. If $p \in S_{n}$ and $a \in Z(p)$ then $(a+\bar{D}) \cap Z\left(p^{\prime}\right) \neq \emptyset$.

Sendov's conjecture is widely regarded as one of the main challenges in the analytic theory of polynomials. Numerous attempts to verify this conjecture have led to over 80 papers, but have met with limited success. We refer to [13], [18] and [19] for surveys of the results on Sendov's conjecture and related questions.

The set $P_{n}$ of monic complex polynomials of degree $n$ may be viewed as a metric space by identifying it with the quotient of $\mathrm{C}^{n}$ by the action of the symmetric group on $n$ elements $\Sigma_{n}$. Indeed, let $\tau: \mathrm{C}^{n} \rightarrow \mathrm{C}^{n} / \Sigma_{n}$ denote the orbit map. Let further $p(z)=\prod_{i=1}^{n}\left(z-z_{i}\right) \in P_{n}, q(z)=\prod_{i=1}^{n}\left(z-\zeta_{i}\right) \in P_{n}$ and set

$$
\Delta(p, q)=\min _{\sigma \in \Sigma_{n}} \max _{1 \leq i \leq n}\left|z_{i}-\zeta_{\sigma(i)}\right| .
$$

Then $\Delta$ is a distance function on $P_{n}$ that induces a structure of compact metric space on the set $S_{n}=\left\{p \in P_{n}: \Delta\left(p, z^{n}\right) \leq 1\right\}=\tau\left(\bar{D}^{n}\right)$. Conjecture 1.1 is

Received July 12, 2004; in revised form May 9, 2005. 
therefore an extremum problem in the closed unit ball in $P_{n}$ for the function $d$ given by

$$
d: S_{n} \rightarrow[0,2], \quad p \mapsto d(p)=\max _{Z \in Z(p)} \min _{w \in Z\left(p^{\prime}\right)}|z-w| .
$$

Note that $d(p)$ is the same as the so-called directed (or oriented) Hausdorff distance from $Z(p)$ to $Z\left(p^{\prime}\right)$ (cf. [18]). Since $d$ is obviously a continuous function it follows by compactness that there exists $p \in S_{n}$ such that $d(p)=$ $\sup _{q \in S_{n}} d(q)$. A polynomial with this property is called extremal for Sendov's conjecture. In 1972 Phelps and Rodriguez proposed the following strengthened form of Sendov's conjecture (cf. [12]):

CONJECTURE 1.2. If $p \in S_{n}$ is extremal for Sendov's conjecture then $p(z)=$ $z^{n}+e^{i \theta}$ for some $\theta \in \mathbf{R}$.

Variational methods are a natural way of dealing with Sendov's conjecture. The relatively few properties known to hold for locally maximal or indeed even extremal polynomials were all deduced by using such methods (see [2], [7], [8], [17]). In this spirit, Miller proposed a slightly more general extremum problem in [7] and [8]. Let $\beta \in \bar{D}$ and denote by $S(n, \beta)$ the set of all polynomials in $S_{n}$ having at least one zero at $\beta$. For $\alpha \in \mathrm{C}$ and $p \in S(n, \beta)$ let

$$
|p|_{\alpha}=\min _{w \in Z\left(p^{\prime}\right)}|\alpha-w|
$$

and define the $\alpha$-critical circle to be the circle with center $\alpha$ and radius $|p|_{\alpha}$. If $p \in S(n, \beta)$ is such that $|p|_{\alpha} \geq|q|_{\alpha}$ for any $q \in S(n, \beta)$ then $p$ is said to be maximal with respect to $\alpha$ in $S(n, \beta)$. Maximal polynomials with respect to $\alpha$ in $S(n, \alpha), \alpha \in \bar{D}$, will be called $\alpha$-maximal for short. A compactness argument again shows that maximal polynomials do exist for any $\alpha \in \mathrm{C}$ and $\beta \in \bar{D}$ ([7, Proposition 2.3]). In 1984 Miller made the following conjecture:

Conjecture 1.3. If $p \in S(n, \beta)$ is maximal with respect to $\alpha$ then $p^{\prime}$ has all its zeros on the $\alpha$-critical circle and $p$ has as many zeros as possible on the unit circle.

For results pertaining to Miller's conjecture we refer to [2], [7], [8] and [17]. The relevance of $\alpha$-maximal polynomials in this context is quite clear. As shown in [7, Proposition 2.4], if $p \in S_{n}$ is an extremal polynomial for Sendov's conjecture then there exists $\alpha \in Z(p)$ such that $p$ is $\alpha$-maximal and $|p|_{\alpha}=d(p)$. Note that $\grave{a}$ priori there may exist $\alpha \in \bar{D}$ such that if $p$ is an $\alpha$-maximal polynomial then $|p|_{\alpha}<d(p)$. The $\alpha$-maximal polynomials that satisfy $|p|_{\alpha}=d(p)$ are particularly interesting since all extremal polynomials are necessarily of this type and they may also provide potential candidates for 
local maxima for the function $d$. It has been known for some time now that if $|\alpha|=1$ then $z^{n}-\alpha^{n}$ is the only $\alpha$-maximal polynomial (cf. [15]). Moreover, this polynomial was shown to be a local maximum for $d$ ([9], [20]). These are actually all the examples of $\alpha$-maximal polynomials known so far, as no such polynomials were found explicitly for $|\alpha|<1$.

The main purpose of this paper is to construct and study new examples of $\alpha$-maximal polynomials. In $\S 2$ we determine all 0 -maximal polynomials (Theorem 2.4) and investigate some of their properties. It turns out that all polynomials of this type satisfy Miller's conjecture as well as the relation $|p|_{0}=d(p)$ (Remark 2.6 and Corollary 2.7). Furthermore, we show that these polynomials are locally extremal for Sendov's problem in $S(n, 0)$, i.e., they are local maxima for the restriction of the function $d$ to $S(n, 0)$ (Proposition 2.9). We conjecture that these are in fact all extremal polynomials for Sendov's conjecture in the class $S(n, 0)$ (Conjecture 2.8).

In $\S 3$ we define and characterize a weaker version of local maximality, namely the notion of linearly inextensible polynomial (Definition 3.2 and Theorems 3.13-3.14). We then investigate the properties of 0-maximal polynomials that are rotations of the polynomial $p(z)=z^{n}+z$. If $n \geq 4$ then $p$ is locally maximal for a large class of variations of its zeros (Proposition 3.20). Moreover, $p$ is locally maximal for the restriction of the function $d$ to $S(n, 0)$ (Proposition 2.9), it is linearly inextensible (Theorem 3.16) and satisfies Conjecture 1.3 (Remark 2.6). These properties and the symmetrical distribution of the zeros and critical points of $p$ seem to suggest that if $n \geq 4$ then $p$ and its rotations could in fact be locally maximal for Sendov's conjecture in the whole class $S_{n}$. The discussion in $\S 3$ shows that first order approximations are not enough for deciding whether this is true or not. In $\$ 4$ we use a second order variational method to prove that despite all appearances $p$ is not locally maximal for Sendov's conjecture. Indeed, Theorems 4.2 and 4.5 show that if $n=4$ or 5 then $p$ is actually a kind of inflection point for the function $d$. We conjecture that the same is true for all higher degrees (Remark 4.6). These results complement those obtained in $\$ 2$ and $\$ 3$ and show quite clearly that first order variational methods cannot provide successful ways of dealing with Sendov's conjecture in its full generality.

AcKNowledgements. We would like to thank A. Meurman and B. Shapiro for stimulating discussions and the anonymous referee for useful suggestions.

\section{Classification of 0-maximal polynomials}

In this section we determine all 0-maximal polynomials (Theorem 2.4) and study some of their properties. We show that these polynomials are local maxima for the function $\left.d\right|_{S(n, 0)}$ (Proposition 2.9) and we conjecture that they are 
in fact all extremal polynomials for Sendov's problem in the class $S(n, 0)$ (Conjecture 2.8).

We start with a few preliminary results, the first of which is well known from the theory of self-inversive polynomials:

LeMma 2.1. Let $p(z)=\sum_{k=0}^{n} a_{k} z^{k}$ be a complex polynomial of degree $n \geq 1$. If all the zeros of $p$ lie on the unit circle then $a_{k} \bar{a}_{0}=a_{n} \bar{a}_{n-k}, 0 \leq k \leq$ $n-1$.

From Lemma 2.1 we deduce the following result.

LeMma 2.2. Let $q$ be a monic complex polynomial of degree $n \geq 2$ and $\alpha \in Z(q)$. If $R>0$ is such that $|w-\alpha|=R$ for any $w \in Z\left(q^{\prime}\right)$ then

$$
n R^{2(n-1)} q^{\prime}(\alpha+z)=q^{\prime}(\alpha) z^{n-1} \overline{q^{\prime}\left(\alpha+R^{2} \bar{z}^{-1}\right)}
$$

for all $z \in \mathrm{C}$, that is,

$$
(n-k-1) ! n R^{2 k} q^{(k+1)}(\alpha)=k ! q^{\prime}(\alpha) \overline{q^{(n-k)}(\alpha)}, \quad 0 \leq k \leq n-1 .
$$

Proof. By assumption, the polynomial

$$
q^{\prime}(\alpha+R z)=\sum_{k=0}^{n-1} \frac{R^{k} q^{(k+1)}(\alpha)}{k !} z^{k}
$$

is of degree at least one and has all its zeros on the unit circle. The identities in (1) follow by applying Lemma 2.1 to the polynomial $q^{\prime}(\alpha+R z)$.

We shall also need the following lemma.

LEMMA 2.3. If $n$ is an integer greater than two then

$$
\left\{x \in[1, n-2]: n^{\frac{2 x}{n-1}}(n-x)-n(x+1)=0\right\}=\left\{\frac{n-1}{2}\right\} .
$$

Proof. The assertion is trivially true for $n=3$ and we may therefore assume that $n \geq 4$. Note that

$$
(-1, n) \ni x \mapsto f(x)=\left(\frac{2 x}{n-1}-1\right) \log n+\log \left(\frac{n-x}{x+1}\right)
$$

is a continuously differentiable function which satisfies

$$
f(0)=f\left(\frac{n-1}{2}\right)=f(n-1)=0 .
$$


It is easily seen that the lemma is in fact equivalent to the following statement:

$$
f(x) \neq 0 \quad \text { for any } \quad x \in[1, n-2] \backslash\left\{\frac{n-1}{2}\right\} .
$$

To prove (2) note that the equation $f^{\prime}(x)=0$ has at most two real roots in the interval $[0, n-1]$ since

$$
f^{\prime}(x)=\frac{2(\log n) x^{2}-2[(n-1) \log n] x+n^{2}-2 n \log n-1}{(n-1)(x+1)(x-n)} .
$$

Thus by Rolle's theorem the equation $f(x)=0$ cannot have more than the three roots listed above in the interval $[0, n-1]$, which establishes (2).

We are now ready to prove the main result of this section:

THEOREM 2.4. The 0 -maximal polynomials of degree $n \geq 2$ are given by:

(i) $z^{2 m}+e^{i 2 \theta} z$ if $n=2 m$ and $m \geq 1$, where $\theta \in \mathbf{R}$.

(ii) $z^{2 m+1}+\lambda e^{i \theta} z^{m+1}+e^{i 2 \theta} z$ if $n=2 m+1$ and $m \geq 1$, where $\lambda, \theta \in \mathbf{R}$ and $|\lambda| \leq \frac{2 \sqrt{2 m+1}}{m+1}$.

Proof. Let $p$ be a 0 -maximal polynomial. Since $p \in S(n, 0)$, we may write

$$
\begin{aligned}
& p(z)=z^{n}+\sum_{k=1}^{n-1} a_{k} z^{k}=z \prod_{i=1}^{n-1}\left(z-z_{i}\right), \quad \text { where }\left|z_{i}\right| \leq 1, \quad 1 \leq i \leq n-1, \\
& p^{\prime}(z)=n z^{n-1}+\sum_{k=1}^{n-1} k a_{k} z^{k-1}=n \prod_{j=1}^{n-1}\left(z-w_{j}\right) .
\end{aligned}
$$

By comparing $p$ with the polynomial $q(z):=z^{n}-z \in S(n, 0)$ we get

$$
\min _{1 \leq j \leq n-1}\left|w_{j}\right|=|p|_{0} \geq|q|_{0}=\left(\frac{1}{n}\right)^{\frac{1}{n-1}},
$$

which combined with the identity $\prod_{i=1}^{n-1} z_{i}=(-1)^{n-1} p^{\prime}(0)=n \prod_{j=1}^{n-1} w_{j}$ yields $\prod_{i=1}^{n-1}\left|z_{i}\right|=n \prod_{j=1}^{n-1}\left|w_{j}\right| \geq 1$. Since $\left|z_{i}\right| \leq 1$ for $1 \leq i \leq n-1$ we deduce that

$$
\left|z_{i}\right|=1, \quad 1 \leq i \leq n-1, \text { and }\left|w_{j}\right|=\left(\frac{1}{n}\right)^{\frac{1}{n-1}}, \quad 1 \leq j \leq n-1 .
$$

It follows in particular that $\left|a_{1}\right|=\left|\prod_{i=1}^{n-1} z_{i}\right|=1$. Hence

$$
a_{1}=e^{i 2 \theta} \quad \text { for some } \quad \theta \in \mathbf{R} .
$$


From (3) and Lemma 2.1 applied to the polynomial $z^{-1} p(z)$ we get

$$
\bar{a}_{n-k}=e^{-i 2 \theta} a_{k+1}, \quad 1 \leq k \leq n-2,
$$

while (3) and Lemma 2.2 applied to the polynomial $p$ with $\alpha=0$ and $R=$ $\left(\frac{1}{n}\right)^{\frac{1}{n-1}}$ imply that

$$
\bar{a}_{n-k}=\frac{n(k+1)}{n-k}\left(\frac{1}{n}\right)^{\frac{2 k}{n-1}} e^{-i 2 \theta} a_{k+1}, \quad 1 \leq k \leq n-2 .
$$

Thus

$$
\left[n^{\frac{2 k}{n-1}}(n-k)-n(k+1)\right] a_{k+1}=0, \quad 1 \leq k \leq n-2,
$$

and then by Lemma 2.3 we get that $a_{k}=0$ for $2 \leq k \leq n-1$ if $n$ is even.

Let now $n=2 m+1, m \geq 1$. In this case Lemma 2.3 and (5) imply that $a_{k}=0$ for $2 \leq k \leq n-1, k \neq m+1$, so that

$$
p(z)=z^{2 m+1}+a_{m+1} z^{m+1}+e^{i 2 \theta} z,
$$

where $\theta$ is as in (4). From (3) and (6) we obtain

$$
\sum_{i=1}^{2 m} z_{i}^{m}+2 m a_{m+1}+e^{i 2 \theta} \sum_{i=1}^{2 m} \bar{z}_{i}^{m}=0 .
$$

Note that by Newton's identities one has actually that

$$
\sum_{i=1}^{2 m} z_{i}^{m}=-m a_{m+1}-\sum_{k=1}^{m-1} a_{m+k+1}\left(\sum_{j=1}^{2 m} z_{j}^{k}\right)=-m a_{m+1} .
$$

We deduce from the last two formulas that $a_{m+1}=e^{i 2 \theta} \bar{a}_{m+1}$, which together with (6) implies that the polynomial $p$ must be of the form

$$
p(z)=z^{2 m+1}+\lambda e^{i \theta} z^{m+1}+e^{i 2 \theta} z, \quad \text { where } \quad \lambda \in \mathbf{R} .
$$

An elementary computation shows that if $\theta, s, t \in \mathbf{R}$ with $t>0$ then the roots of the equation $x^{2}+s e^{i \theta} x+t e^{i 2 \theta}=0$ cannot have the same absolute value unless $s^{2} \leq 4 t$. By (3) all the zeros of the polynomial

$$
p^{\prime}(z)=(2 m+1) z^{2 m}+(m+1) \lambda e^{i \theta} z^{m}+e^{i 2 \theta}
$$

have the same absolute value. Now using the substitution $x=z^{m}$ and the above-mentioned result on the roots of second degree equations we see that 
this cannot happen unless $|\lambda| \leq \frac{2 \sqrt{2 m+1}}{m+1}$. To summarize, we have shown that if $p \in S(n, 0)$ then $|p|_{0}<\left(\frac{1}{n}\right)^{\frac{1}{n-1}}$ unless $p$ is of the form (i) or (ii) according to the parity of $n$. On the other hand, it is easily checked that a polynomial of the form (i) or (ii) satisfies $|p|_{0}=\left(\frac{1}{n}\right)^{\frac{1}{n-1}}$, which completes the proof of the theorem.

REMARK 2.5. The estimate $|p|_{0} \leq\left(\frac{1}{n}\right)^{\frac{1}{n-1}}$ for $p \in S(n, 0)$ appears also in [1, Lemma 4] but the cases of equality are not dealt with in loc. cit.

Remark 2.6. Let $p$ be a 0 -maximal polynomial of degree $n \geq 2$. By Theorem 2.4 all the critical points of $p$ lie on the 0 -critical circle $|z|=\left(\frac{1}{n}\right)^{\frac{1}{n-1}}$ and all the zeros of $p$ except 0 lie on the unit circle. Thus, all 0 -maximal polynomials satisfy Miller's conjecture (Conjecture 1.3). Note also that if $p$ is 0 -maximal then it has only simple zeros. The same is true for $p^{\prime}$ except when $n$ is odd and $p$ is of the form (ii) with $|\lambda|=\frac{4 \sqrt{n}}{n+1}$, in which case $p^{\prime}$ has $\frac{n-1}{2}$ distinct zeros each of multiplicity two.

Corollary 2.7. If $p$ is a 0-maximal polynomial of degree $n \geq 2$ then $d(p)=|p|_{0}$.

Proof. Let $z \in Z(p) \backslash\{0\}$, so that $|z|=1$ by Remark 2.6. Denote by $H_{z}$ the closed half-plane that contains $z$ and is bounded by the line passing through $\frac{z}{2}$ which is orthogonal to the segment $[0, z]$. Since $p(z)=0=p(0)$ it follows from a well-known consequence of the Grace-Heawood theorem that there exists $w \in Z\left(p^{\prime}\right) \cap H_{z}$ (see, e.g., the supplement to Theorem 4.3.1 in [13]). By Remark 2.6 again one has that $|w|=|p|_{0}=\left(\frac{1}{n}\right)^{\frac{1}{n-1}} \geq \frac{1}{2}$. It is then geometrically clear that $|z-w| \leq|p|_{0}$ and thus $\min _{\omega \in Z\left(p^{\prime}\right)}|z-\omega| \leq|p|_{0}$, which proves the desired result.

Theorem 2.4 and Corollary 2.7 do not automatically imply that 0-maximal polynomials are global maxima for the restriction of the function $d$ to the subset $S(n, 0)$ of $S_{n}$. For this one would have to show that $d(p) \leq\left(\frac{1}{n}\right)^{\frac{1}{n-1}}$ whenever $p \in S(n, 0)$, which we actually conjecture to be true:

CONJECTURE 2.8. If $p \in S(n, 0)$ then $d(p) \leq\left(\frac{1}{n}\right)^{\frac{1}{n-1}}$ and equality occurs if and only if $p$ is as in Theorem 2.4.

In other words, Conjecture 2.8 asserts that Theorem 2.4 gives all extremal polynomials for Sendov's problem restricted to the class $S(n, 0)$. We should point out that the best estimate known so far for $p \in S(n, 0)$ is $d(p)<$ 1 (see [13, Theorem 7.3.6]). Our next result shows that the polynomials in Theorem 2.4 are locally maximal for Sendov's problem in the class $S(n, 0)$, so that Conjecture 2.8 is at least locally valid. 
Proposition 2.9. If $p$ is a polynomial as in Theorem 2.4 there exists $\varepsilon>0$ such that whenever $q \in S(n, 0)$ and $\Delta(q, p)<\varepsilon$ one has $d(q) \leq\left(\frac{1}{n}\right)^{\frac{1}{n-1}}$. Moreover, the latter inequality is strict unless $q$ is itself a polynomial as in Theorem 2.4.

Proof. The statement is trivially true if $n=2$ and so we may assume that $n \geq 3$. If $p$ is a polynomial as in Theorem 2.4 then the proof of Corollary 2.7 shows that $|p|_{z}<|p|_{0}=d(p)$ for $z \in Z(p) \backslash\{0\}$. Hence there exists $\varepsilon=$ $\varepsilon(p)>0$ such that $|q|_{\zeta}<|q|_{0}=d(q)$ for $\zeta \in Z(q) \backslash\{0\}$ whenever $q \in$ $S(n, 0)$ and $\Delta(q, p)<\varepsilon$. The result is now a consequence of Theorem 2.4.

REMARK 2.10. It is not difficult to see that that $\max _{p \in S(n, 1)}|p|_{1}=1$ (cf. [15]). On the other hand, Theorem 2.4 shows that $\max _{p \in S(n, 0)}|p|_{0}$ increases to 1 as $n \rightarrow \infty$. It would be interesting to know whether there exist $\alpha \in D$ and $c \in(0,1)$ such that $\max _{p \in S(n, \alpha)}|p|_{\alpha} \leq c$ for any $n \geq 2$.

To end this section let us point out that Theorem 2.4 solves in fact the following more general extremum problem concerning the distribution of zeros and critical points of complex polynomials:

Problem 2.11. Let $n \geq 2, a \in \mathrm{C}$, and $R>0$. Find the largest constant $\rho:=\rho(a, n, R)$ with the property that for any complex polynomial $p$ of degree $n$ satisfying $p(a)=0$ and $\min _{w \in Z\left(p^{\prime}\right)}|w-a| \geq R$ one has $\max _{z \in Z(p)}|z-a| \geq$ $\rho$.

It is easy to see that $\rho$ is invariant under translations in the complex plane so that it does not depend on $a$. Moreover, the Gauss-Lucas theorem clearly implies that $\rho>R$. On the other hand, by considering $p(z)=(z-a)^{n}+$ $n R^{n-1}(z-a)$ we see that $\rho \leq R n^{\frac{1}{n-1}}$. Essentially the same computations as in the proof of Theorem 2.4 show that in fact $\rho=R n^{\frac{1}{n-1}}$ and the extremal cases are as follows.

THEOREM 2.12. In the above notations one has $\rho=R n^{\frac{1}{n-1}}$ and this value is attained only for the following polynomials:

(i) $(z-a)^{2 m}+2 m R^{2 m-1} e^{i \theta}(z-a)$ if $n=2 m$ and $m \geq 1$, where $\theta \in \mathbf{R}$.

(ii) $(z-a)^{2 m+1}+\lambda \sqrt{2 m+1} R^{m} e^{i \theta}(z-a)^{m+1}+(2 m+1) R^{2 m} e^{i 2 \theta}(z-a)$ if $n=2 m+1$ and $m \geq 1$, where $\lambda, \theta \in \mathrm{R}$ and $|\lambda| \leq \frac{2 \sqrt{2 m+1}}{m+1}$. 


\section{First order expansions and linearly inextensible polynomials}

In the previous section we have already mentioned the notion of local maximum for the Sendov problem. A formal definition of this notion is as follows.

Definition 3.1. Given $\varepsilon>0$ and $p \in S_{n}$ we define the $\varepsilon$-neighborhood of $p$ to be $V_{\varepsilon}(p)=\left\{q \in S_{n}: \Delta(q, p)<\varepsilon\right\}$. A polynomial $p \in S_{n}$ is called locally maximal for Sendov's conjecture if it is a local maximum for the function $d$, i.e., there exists $\varepsilon>0$ such that $d(q) \leq d(p)$ for any $q \in V_{\varepsilon}(p)$.

We shall now define and study a weaker version of this notion.

Definition 3.2. A polynomial $p \in S_{n}$ is linearly inextensible if there exist $\varepsilon>0$ and constants $C \geq 0, \kappa>0$ depending only on $p$ such that $d(q) \leq d(p)+C \Delta(q, p)^{1+\kappa}$ for all $q \in V_{\varepsilon}(p)$. Otherwise $p$ is said to be linearly extensible.

Clearly, a locally maximal polynomial is necessarily linearly inextensible while a linearly extensible polynomial cannot be locally maximal. Necessary criteria for local maximality in certain generic cases were established in [2] and [7].

Definition 3.2 is motivated mainly by two facts. On the one hand, a close examination of the aforementioned criteria shows that they are in fact both necessary and sufficient conditions for linear inextensibility in the generic cases under consideration (Theorems 3.13-3.14). On the other hand, linear inextensibility can be decided by means of first order expansions while local maximality may require higher order approximations. The examples below show that these are actually crucial differences between the notions in Definitions 3.1 and 3.2 and that first order variational methods are clearly not enough for studying local maxima for Sendov's conjecture.

We need some further preliminaries. Let us first recall [7, Definition 2.19]:

Definition 3.3. A complex $m \times n$ matrix $M=\left(m_{i j}\right)$ is positively singular if there exist $\mu_{1}, \ldots, \mu_{m} \geq 0$, not all 0 , so that $\sum_{i=1}^{m} \mu_{i} m_{i j}=0$ for $1 \leq j \leq n$.

Given a vector $\mathbf{y}=\left(y_{1}, \ldots, y_{n}\right) \in \mathrm{C}^{n}$ we shall formally write $\mathfrak{R}(\mathbf{y})>0$ whenever the inequalities $\Re\left(y_{i}\right)>0,1 \leq i \leq n$, are satisfied.

TheOREM 3.4. Let $M$ be a complex $m \times n$ matrix and let $\mathbf{x} \in \mathrm{C}^{n}$ denote a vector of complex unknowns. The system $\Re(M \mathbf{x})>0$ is solvable if and only if $M$ is not positively singular.

REMARK 3.5. Theorem 3.4 is a generalization of what is usually called the fundamental theorem of linear programming, see e.g. [14, Theorem 22.2] and [7, Lemma 2.20]. 
Notation 3.6. For $p \in S_{n}$ we shall use the following generic notations:

$$
\begin{gathered}
p(z)=\prod_{i=1}^{n}\left(z-z_{i}\right), \quad a=z_{1}, \quad|p|_{a}=d(p), \quad p^{\prime}(z)=n \prod_{j=1}^{n-1}\left(z-w_{j}\right), \\
\text { (7) } \quad r=r(a) \in\{1, \ldots, n-1\} \text { and } 1 \leq s_{1}<\ldots<s_{m} \leq n \text { are such that } \\
\left|w_{j}-a\right|=|p|_{a} \text { for } 1 \leq j \leq r, \quad\left|w_{j}-a\right|>|p|_{a} \text { for } j \geq r+1 \\
\left|z_{s_{k}}\right|=1 \text { for } 1 \leq k \leq m, \quad\left|z_{i}\right|<1 \text { for } i \in\{1, \ldots, n\} \backslash\left\{s_{1}, \ldots, s_{m}\right\}
\end{gathered}
$$

The following result combines Lemmas 2.1 and 2.3 in [2].

LEMMA 3.7. If $p$ is as in (7) and has only simple zeros there exist neighborhoods $U, V \subset \mathrm{C}^{n}$ of the points $u=\left(a, w_{1}, \ldots, w_{n-1}\right)$ and $v=\left(a, z_{2}, \ldots, z_{n}\right)$, respectively, such that

$$
U \ni\left(\alpha, \omega_{1}, \ldots, \omega_{n-1}\right) \mapsto\left(\zeta_{1}, \zeta_{2}, \ldots, \zeta_{n}\right) \in V
$$

is an analytic function, where $\zeta_{2}, \ldots, \zeta_{n}$ are the (simple) zeros different from $\alpha$ of the polynomial $n \int_{\alpha}^{z} \prod_{j=1}^{n-1}\left(w-\omega_{j}\right) d w$ and $\zeta_{1}=\zeta_{1}\left(\alpha, \omega_{1}, \ldots, \omega_{n-1}\right) \equiv \alpha$. Moreover, one has

$$
\left.\frac{\partial \zeta_{k}}{\partial \omega_{l}}\right|_{u}=\frac{1}{p^{\prime}\left(z_{k}\right)} \int_{a}^{z_{k}} \frac{p^{\prime}(w)}{w-w_{l}} d w, \quad 2 \leq k \leq n, \quad 1 \leq l \leq n-1 .
$$

From Lemma 3.7 and the inverse function theorem one immediately gets:

LEMMA 3.8. Let $p$ be as in (7) and assume that both $p$ and $p^{\prime}$ have simple zeros. Then in the notations of Lemma 3.7 the functions $\omega_{1}, \ldots, \omega_{n-1}$ are locally analytic in $\zeta_{1}, \zeta_{2}, \ldots, \zeta_{n}$ and

$$
\left.\frac{\partial \omega_{j}}{\partial \zeta_{i}}\right|_{v}=-\frac{p\left(w_{j}\right)}{\left(w_{j}-z_{i}\right)^{2} p^{\prime \prime}\left(w_{j}\right)}, \quad 1 \leq i \leq n, \quad 1 \leq j \leq n-1 .
$$

REMARK 3.9. An interesting byproduct of Lemmas 3.7 and 3.8 is the following "generalized Cauchy determinant" property for complex polynomials: let $p$ be a complex polynomial of degree $n \geq 2$ such that both $p$ and $p^{\prime}$ have simple zeros, set $Z(p)=\left\{z_{1}, \ldots, z_{n}\right\}, Z\left(\overline{p^{\prime}}\right)=\left\{w_{1}, \ldots, w_{n-1}\right\}$ and define an $(n-1) \times(n-1)$ matrix $B(p)=\left(\left(z_{i}-w_{j}\right)^{-2}\right), 1 \leq i, j \leq n-1$. Then $\operatorname{det}(B(p)) \neq 0$ (cf. [3, Theorem 1.3]).

Notation 3.10. Denote by $\|\cdot\|$ the norm on $C^{n}$ given by $\|\mathbf{x}\|=\max _{1 \leq i \leq n}$ $\left|x_{i}\right|$ for $\mathbf{x}=\left(x_{1}, \ldots, x_{n}\right) \in \mathrm{C}^{n}$. Given $\mathbf{e}=\left(\varepsilon_{1}, \ldots, \varepsilon_{n}\right) \in \mathrm{C}^{n}$ and a polynomial 
$p \in S_{n}$ as in (7) define $z_{i}(\mathbf{e})=z_{i}+\varepsilon_{i}, 1 \leq i \leq n$, and set

$$
p_{\mathbf{e}}(z)=\prod_{i=1}^{n}\left(z-z_{i}(\mathbf{e})\right), \quad p_{\mathbf{e}}^{\prime}(z)=n \prod_{j=1}^{n-1}\left(z-w_{j}(\mathbf{e})\right) .
$$

Note that $z_{i}(\mathbf{0})=z_{i}, 1 \leq i \leq n, w_{j}(\mathbf{0})=w_{j}, 1 \leq j \leq n-1$, and that if $p$ has simple zeros then $p_{\mathbf{e}}$ has simple zeros and $\Delta\left(p_{\mathbf{e}}, p\right)=\|\mathbf{e}\|$ for all $\mathbf{e} \in \mathrm{C}^{n}$ with $\|\mathbf{e}\|$ sufficiently small. Clearly, $p_{\mathbf{e}} \in S_{n}$ if and only if $\left|z_{i}+\varepsilon_{i}\right| \leq 1,1 \leq i \leq n$.

Lemma 3.8 and straightforward computations yield the following result.

Proposition 3.11. Let $p$ be as in (7) and such that both $p$ and $p^{\prime}$ have simple zeros. For any $\mathbf{e}=\left(\varepsilon_{1}, \ldots, \varepsilon_{n}\right) \in \mathrm{C}^{n}$ with $\|\mathbf{e}\|$ sufficiently small one has

$$
\left|w_{j}(\mathbf{e})-z_{1}(\mathbf{e})\right|=|p|_{a}\left[1+\sum_{i=1}^{n} \Re\left(\alpha_{i}\left(w_{j} ; a\right) \varepsilon_{i}\right)+\mathcal{O}\left(\|\mathbf{e}\|^{2}\right)\right], \quad 1 \leq j \leq r(a),
$$

where

$$
\begin{aligned}
& \alpha_{1}\left(w_{j} ; a\right)=-\frac{1}{w_{j}-a}\left[1+\frac{p\left(w_{j}\right)}{\left(w_{j}-a\right)^{2} p^{\prime \prime}\left(w_{j}\right)}\right], \\
& \alpha_{i}\left(w_{j} ; a\right)=-\frac{p\left(w_{j}\right)}{\left(w_{j}-a\right)\left(w_{j}-z_{i}\right)^{2} p^{\prime \prime}\left(w_{j}\right)}, \quad 2 \leq i \leq n .
\end{aligned}
$$

Notation 3.12. Given a polynomial $p$ as in (7) such that both $p$ and $p^{\prime}$ have simple zeros we define an $(r(a)+m) \times n$ matrix $A(p ; a)=\left(a_{i j}\right)$ by setting

$$
a_{i j}= \begin{cases}\alpha_{j}\left(w_{i} ; a\right) & \text { if } 1 \leq i \leq r(a), 1 \leq j \leq n, \\ -\bar{z}_{s_{k}} & \text { if } i=r(a)+k \text { and } j=s_{k}, 1 \leq k \leq m, \\ 0 & \text { otherwise. }\end{cases}
$$

We can now prove a necessary and sufficient criterion for linear inextensibility.

Theorem 3.13. Let $p \in S_{n}$ be a polynomial as in (7) with $n \geq 3$. Assume that both $p$ and $p^{\prime}$ have simple zeros and that $|p|_{z_{i}}<d(p)$ for $2 \leq i \leq n$. Then $p$ is linearly inextensible if and only if $A(p ; a)$ is positively singular.

Proof. Suppose that $p$ is linearly inextensible and that there exists $\mathbf{e} \in \mathrm{C}^{n}$ such that $\Re(A(p ; a) \mathbf{e})>0$. Let $t>0$ and construct the polynomial $p_{t \mathbf{e}}(z)$ 
as in (8). Then $p_{t \mathrm{e}}$ has simple zeros and $\Delta\left(p_{t \mathbf{e}}, p\right)=t\|\mathbf{e}\|$ for all sufficiently small $t$. By Proposition 3.11 there exists a constant $\lambda>0$ depending only on $p$ (and $\mathbf{e}$ ) such that

$$
d\left(p_{t \mathbf{e}}\right)=\left|p_{t \mathbf{e}}\right|_{z_{1}(t \mathbf{e})}=\min _{1 \leq j \leq r(a)}\left|w_{j}(t \mathbf{e})-z_{1}(t \mathbf{e})\right|>|p|_{a}(1+\lambda t)
$$

for all small $t>0$, which contradicts the fact that $p$ is linearly inextensible. Hence the system $\Re(A(p ; a) \mathbf{x})>0, x \in \mathrm{C}^{n}$, cannot be solvable and so by Theorem $3.4 A(p ; a)$ must be positively singular.

To establish the sufficiency part note first that if $z, \varepsilon \in \mathrm{C}$ are such that $\varepsilon \neq 0,|z|=1$ and $|z+\varepsilon| \leq 1$ then $\Re(\varepsilon \bar{z})<0$. Recall (9), let $1 \leq k_{1}<$ $\ldots<k_{l} \leq m$ be an ordered $l$-tuple of indices, where $l \leq m$, and denote by $A(p ; a)\left[k_{1}, \ldots, k_{l}\right]$ the $(r(a)+m-l) \times(n-l)$ matrix obtained by deleting rows $r(a)+k_{1}, \ldots, r(a)+k_{l}$ and columns $s_{k_{1}}, \ldots, s_{k_{l}}$ from $A(p ; a)$. Suppose now that $A(p ; a)$ is positively singular. Then so is any matrix of the form $A(p ; a)\left[k_{1}, \ldots, k_{l}\right]$ provided it is not the empty matrix, which can only occur if $l=m=n$.

Let $\mathbf{e}=\left(\varepsilon_{1}, \ldots, \varepsilon_{n}\right) \in \mathbf{C}^{n} \backslash\{\mathbf{0}\}$ be such that $\|\mathbf{e}\|$ is arbitrarily small and consider the polynomial $p_{\mathbf{e}}(z)$ as in (8), on which we impose the condition $p_{\mathbf{e}} \in S_{n}$. Set

$$
K(\mathbf{e})=\left\{k_{1}(\mathbf{e}), \ldots, k_{l}(\mathbf{e})\right\}:=\left\{i \in\{1, \ldots, m\}: \varepsilon_{s_{i}}=0\right\},
$$

so that $l \leq n-1$ since $\mathbf{e} \neq \mathbf{0}$. Assuming as we may that $k_{1}(\mathbf{e})<\ldots<k_{l}(\mathbf{e})$ let us construct the corresponding (non-empty) matrix $A(p ; a)\left[k_{1}(\mathbf{e}), \ldots, k_{l}(\mathbf{e})\right]$ as above, where it is understood that $A(p ; a)\left[k_{1}(\mathbf{e}), \ldots, k_{l}(\mathbf{e})\right]=A(p ; a)$ if $K(\mathbf{e})=\emptyset$. Since $\Re\left(\varepsilon_{s_{i}} \bar{z}_{s_{i}}\right)<0$ for $i \in\{1, \ldots, m\} \backslash K(\mathbf{e})$ and $A(p ; a)\left[k_{1}(\mathbf{e})\right.$, $\left.\ldots, k_{l}(\mathbf{e})\right]$ is positively singular it follows from (9) and Theorem 3.4 that there exists $j \in\{1, \ldots, r(a)\}$ such that

$$
\sum_{i=1}^{n} \Re\left(\alpha_{i}\left(w_{j} ; a\right) \varepsilon_{i}\right) \leq 0 .
$$

Proposition 3.11 implies that there exists a non-negative constant $C$ depending only on $p$ such that

$$
d\left(p_{\mathbf{e}}\right)=\left|p_{\mathbf{e}}\right|_{z_{1}(\mathbf{e})}=\min _{1 \leq j \leq r(a)}\left|w_{j}(\mathbf{e})-z_{1}(\mathbf{e})\right| \leq|p|_{a}\left(1+C\|\mathbf{e}\|^{2}\right),
$$

which shows that $p$ is linearly inextensible.

The following generalization of Theorem 3.13 is obtained in similar fashion and we therefore state it without proof. 
THEOREM 3.14. Let $p \in S_{n}$ be as in (7) with $n \geq 3$ and assume that both $p$ and $p^{\prime}$ have simple zeros. Suppose further that the $z_{i}$ 's are labeled so that $|p|_{z_{i}}=d(p)$ for $1 \leq i \leq t$ and $|p|_{z_{i}}<d(p)$ for $i \geq t+1$, where $t \in\{1, \ldots, n\}$. Then $p$ is linearly inextensible if and only if $A\left(p ; z_{i}\right), 1 \leq i \leq t$, are positively singular matrices.

REMARK 3.15. Let

$$
\begin{aligned}
& \hat{S}_{n}=\left\{p \in S_{n}: p \text { and } p^{\prime} \text { have simple zeros }\right\} \quad \text { and } \\
& \tilde{S}_{n}=\left\{p \in S_{n}: \text { all zeros of } p \text { on the unit circle are simple }\right\},
\end{aligned}
$$

so that $\hat{S}_{n} \subsetneq \tilde{S}_{n}$. The necessity part in Theorem 3.13 viewed as a necessary condition for local maximality in the class $\hat{S}_{n}$ was established in [7]. A necessary criterion for local maximality in the larger class $\tilde{S}_{n}$ was obtained in [2]. It is not difficult to see that the latter actually gives necessary and essentially also sufficient conditions for linear inextensibility in the class $\tilde{S}_{n}$.

The 0 -maximal polynomials given in Theorem 2.4 provide natural examples of linearly inextensible polynomials of arbitrary degree:

Theorem 3.16. Let $\theta \in \mathrm{R}, n \geq 3$, and $p(z)=z^{n}+e^{i \theta} z$. Then $|p|_{0}=d(p)$, $|p|_{\zeta}<d(p)$ for $\zeta \in Z(p) \backslash\{0\}$ and $p$ is linearly inextensible.

Proof. It is enough to prove the statement for the polynomial $p(z)=z^{n}-z$ since $z^{n}+e^{i \theta} z=e^{-i n \alpha} p\left(e^{i \alpha} z\right)$, where $\alpha=\frac{\pi-\theta}{n-1}$. Recall the notations of (7) and set

$$
\begin{gathered}
(a=) z_{1}=0, \quad z_{j}=e^{\frac{2 \pi i(j-2)}{n-1}}, \quad 2 \leq j \leq n, \\
w_{k}=\left(\frac{1}{n}\right)^{\frac{1}{n-1}} e^{\frac{2 \pi i(k-1)}{n-1}}, \quad 1 \leq k \leq n-1 .
\end{gathered}
$$

It is easy to check that $|p|_{0}=d(p),|p|_{\zeta}<d(p)$ for $\zeta \in Z(p) \backslash\{0\}$ and $r(0)=n-1$, so that the matrix $A(p ; a)$ defined in (9) is actually a $2(n-1) \times n$ matrix. Proposition 3.11 and elementary computations yield

$$
\begin{aligned}
& \alpha_{1}\left(w_{i} ; a\right)=-\frac{n-1}{n w_{i}}, \\
& \alpha_{j}\left(w_{i} ; a\right)=\frac{w_{i}}{n\left(w_{i}-z_{j}\right)^{2}}, \quad 1 \leq i \leq n-1,2 \leq j \leq n .
\end{aligned}
$$


Using the fact that for $z \in \mathrm{C} \backslash Z\left(p^{\prime}\right)$ one has the well-known identities

$$
\begin{aligned}
& \frac{p^{\prime \prime}(z)}{p^{\prime}(z)}=\sum_{i=1}^{n-1} \frac{1}{z-w_{i}} \quad \text { and } \\
& \frac{p^{(3)}(z) p^{\prime}(z)-\left(p^{\prime \prime}(z)\right)^{2}}{\left(p^{\prime}(z)\right)^{2}}=\frac{d}{d z}\left[\frac{p^{\prime \prime}(z)}{p^{\prime}(z)}\right]=-\sum_{i=1}^{n-1} \frac{1}{\left(z-w_{i}\right)^{2}}
\end{aligned}
$$

one can show that if $2 \leq j \leq n$ then

$$
\begin{aligned}
\sum_{i=1}^{n-1} \frac{w_{i}}{\left(w_{i}-z_{j}\right)^{2}} & =\sum_{i=1}^{n-1} \frac{1}{w_{i}-z_{j}}+z_{j} \sum_{i=1}^{n-1} \frac{1}{\left(w_{i}-z_{j}\right)^{2}} \\
& =-\frac{p^{\prime \prime}\left(z_{j}\right)}{p^{\prime}\left(z_{j}\right)}+z_{j}\left[\frac{\left(p^{\prime \prime}\left(z_{j}\right)\right)^{2}-p^{(3)}\left(z_{j}\right) p^{\prime}\left(z_{j}\right)}{\left(p^{\prime}\left(z_{j}\right)\right)^{2}}\right]=\frac{n}{z_{j}} .
\end{aligned}
$$

It follows that

$$
\begin{aligned}
& \sum_{i=1}^{n-1} \alpha_{1}\left(w_{i} ; a\right)=-\frac{n-1}{n} \sum_{i=1}^{n-1} \frac{1}{w_{i}}=0, \\
& \sum_{i=1}^{n-1} \alpha_{j}\left(w_{i} ; a\right)-\bar{z}_{j}=\frac{1}{z_{j}}-\bar{z}_{j}=0, \quad 2 \leq j \leq n,
\end{aligned}
$$

which shows that $A(p ; a)$ is a positively singular matrix. By Theorem 3.13 the polynomial $p(z)=z^{n}-z$ cannot be linearly extensible.

We can now give a concrete example of a linearly inextensible polynomial which is not a local maximum for Sendov's conjecture.

Lemma 3.17. If $\theta \in \mathrm{R}$ then $p(z)=z^{3}+e^{i \theta} z$ is not a local maximum for $d$.

Proof. Note that $d(p)=|p|_{0}=\frac{1}{\sqrt{3}}$ and that by a rotation we may assume that $p(z)=z^{3}-z$. Let $t \in[0,1]$ and set

$$
q_{t}(z)=(z-i t)\left(z^{2}-1\right) .
$$

Then $q_{t} \in S_{3}$ and elementary computations show that for all small $t>0$ one has

$$
d\left(q_{t}\right)=\left|q_{t}\right|_{i t}=\sqrt{\frac{1+t^{2}}{3}}>d(p),
$$

which proves the lemma. 
Remark 3.18. In $\S 4$ we shall construct explicit examples of linearly inextensible polynomials of degree greater than 3 which are not local maxima for $d$.

In the remainder of this section we establish further properties of the 0maximal and linearly inextensible polynomials of the form $z^{n}-z$ with $n \geq 4$.

Notation 3.19. Recall the notations used in the proof of Theorem 3.16 and set

$$
\begin{aligned}
& p(z)=z^{n}-z=\prod_{j=1}^{n}\left(z-z_{j}\right) \text { and } p^{\prime}(z)=n \prod_{k=1}^{n-1}\left(z-w_{k}\right), \text { where } z_{1}=0, \\
& z_{j}=e^{\frac{2 \pi i(j-2)}{n-1}}, 2 \leq j \leq n, \text { and } w_{k}=\left(\frac{1}{n}\right)^{\frac{1}{n-1}} e^{\frac{2 \pi i(k-1)}{n-1}}, 1 \leq k \leq n-1 .
\end{aligned}
$$

Let $\kappa$ be a fixed positive number and $\mathbf{e}=\left(\varepsilon_{1}, \ldots, \varepsilon_{n}\right) \in \mathrm{C}^{n}$ be such that $\left|\varepsilon_{j}\right| \leq$ $\left|\varepsilon_{1}\right|^{1+\kappa}$ for $2 \leq j \leq n$ and let $p_{\mathbf{e}}$ be as in (8), that is, $p_{\mathbf{e}}(z)=\prod_{j=1}^{n}\left(z-\left(z_{j}+\varepsilon_{j}\right)\right)$.

Using the above notations we can show the following result:

PROPOSITION 3.20. If $n \geq 4$ then for all sufficiently small $\left|\varepsilon_{1}\right|$ one has

$$
\min _{\omega \in Z\left(p_{\mathbf{e}}^{\prime}\right)}\left|z_{1}+\varepsilon_{1}-\omega\right| \leq \min _{\omega \in Z\left(p^{\prime}\right)}\left|z_{1}-\omega\right|-\left[\cos \left(\frac{\pi}{n-1}\right)\right]\left|\varepsilon_{1}\right| .
$$

Thus, if $\varepsilon_{j}=0$ for $2 \leq j \leq n$ or, more generally, if $\left|z_{j}+\varepsilon_{j}\right| \leq 1$ for $2 \leq j \leq n$ then for all sufficiently small $\left|\varepsilon_{1}\right|>0$ one has $p_{\mathbf{e}} \in S_{n}$ and $d\left(p_{\mathbf{e}}\right)<d(p)$.

PROOF. Note that $\left|w_{k}\right|=\left(\frac{1}{n}\right)^{\frac{1}{n-1}}=|p|_{z_{1}}=d(p), 1 \leq k \leq n-1$, and denote the zeros of $p_{\mathbf{e}}^{\prime}(z)$ by $\omega_{k}=\omega_{k}\left(\varepsilon_{1}, \ldots, \varepsilon_{n}\right), 1 \leq k \leq n-1$. We assume that these are labeled so that $\omega_{k}(0, \ldots, 0)=w_{k}, 1 \leq k \leq n-1$. It follows from (10) that if $\varepsilon_{1} \neq 0$ then there exists $j \in\{1, \ldots, n-1\}$ such that

(11) $\left|\arg \varepsilon_{1}-\arg w_{j}\right| \leq \frac{\pi}{n-1}$, so that $\Re\left(\frac{\varepsilon_{1}}{w_{j}}\right) \geq \frac{\left|\varepsilon_{1}\right|}{d(p)} \cos \left(\frac{\pi}{n-1}\right)$.

Now using Lemma 3.8 with $v=\left(0, z_{2}, \ldots, z_{n}\right)$ and the computations in the proof of Theorem 3.16 together with the assumption $\left|\varepsilon_{i}\right| \leq\left|\varepsilon_{1}\right|^{1+\kappa}, 2 \leq i \leq n$, we get

$$
\begin{aligned}
\left|\omega_{j}\left(\varepsilon_{1}, \ldots, \varepsilon_{n}\right)-\varepsilon_{1}\right| & =\left|w_{j}-\varepsilon_{1}+\sum_{i=1}^{n} \frac{\partial \omega_{j}}{\partial \zeta_{i}}\right|_{v} \varepsilon_{i}+\mathcal{O}\left(\left|\varepsilon_{1}\right|^{2}\right) \mid \\
& =\left|w_{j}\right|-\frac{n-1}{n} \Re\left(\frac{\varepsilon_{1}}{w_{j}}\right)+\mathscr{O}\left(\left|\varepsilon_{1}\right|^{1+\kappa^{\prime}}\right),
\end{aligned}
$$


where $\kappa^{\prime}=\min (1, \kappa)$. It is not difficult to see that if $n \geq 4$ then $d(p)=$ $\left(\frac{1}{n}\right)^{\frac{1}{n-1}}<1-\frac{1}{n}$. From (11)-(12) and the latter inequality it follows that if $n \geq 4$ then for all small $\left|\varepsilon_{1}\right|$ one has

$$
\begin{aligned}
\left|\omega_{j}\left(\varepsilon_{1}, \ldots, \varepsilon_{n}\right)-\varepsilon_{1}\right| & \leq\left|w_{j}\right|-\left[\frac{n-1}{n d(p)} \cos \left(\frac{\pi}{n-1}\right)\right]\left|\varepsilon_{1}\right|+\mathscr{O}\left(\left|\varepsilon_{1}\right|^{1+\kappa^{\prime}}\right) \\
& \leq d(p)-\left[\cos \left(\frac{\pi}{n-1}\right)\right]\left|\varepsilon_{1}\right|,
\end{aligned}
$$

which proves the proposition.

As already noted in Remark 2.10, if $p \in S_{n}$ and $\zeta \in Z(p)$ is such that $|\zeta|=1$ then $|p|_{\zeta} \leq 1$. It is therefore natural to ask whether the following holds:

Problem 3.21. Let $p(z)=\left(z-z_{1}\right) q(z) \in S_{n}$, where $\left|z_{1}\right|<1$ and $q \in S_{n-1}$. Does there exist $\zeta \in \mathrm{C}$ with $|\zeta|=1$ such that $|p|_{z_{1}} \leq|r|_{\zeta}$, where $r(z)=(z-\zeta) q(z)$ ?

In other words, is it always possible to increase the Sendov distance for a given zero of $p$ in the unit disk by "pushing" this zero to the boundary while keeping the other zeros of $p$ fixed? The 0-maximal polynomials of the form $z^{n}-z$ show that such a property cannot hold in general:

PRoposition 3.22. If $n \geq 5$ there exist $z_{1} \in D$ and $q \in S_{n-1}$ such that for any $\zeta \in \mathrm{C}$ with $|\zeta|=1$ one has $|r|_{\zeta}<|p|_{z_{1}}$, where $p(z)=\left(z-z_{1}\right) q(z)$ and $r(z)=(z-\zeta) q(z)$.

Proof. Let $\zeta \in \mathrm{C}$ be such that $|\zeta|=1$ and set $z_{1}=0$ and $q(z)=z^{n-1}-1$, so that $p(z)=z^{n}-z$ and $r(z)=(z-\zeta)\left(z^{n-1}-1\right)$. Then for any $\omega \in Z\left(p^{\prime}\right)$ one has $\left|z_{1}-\omega\right|=\left(\frac{1}{n}\right)^{\frac{1}{n-1}}$, so that

$$
|p|_{z_{1}}=\min _{\omega \in Z\left(p^{\prime}\right)}\left|z_{1}-\omega\right|=\left(\frac{1}{n}\right)^{\frac{1}{n-1}} .
$$

Since $|\zeta|=1$ and $Z(q)=\left\{e^{\frac{2 k \pi i}{n-1}}: 0 \leq k \leq n-2\right\}$ one gets from either the Schur-Szegö composition theorem ([13, Theorem 3.4.1d]) or the GraceHeawood theorem ([13, Theorem 4.3.1]) that

$$
|r|_{\zeta}=\min _{\omega \in Z\left(r^{\prime}\right)}|\zeta-\omega| \leq \frac{\min _{\alpha \in Z(q)}|\zeta-\alpha|}{2 \sin (\pi / n)} \leq \frac{\sin (\pi / 2(n-1))}{\sin (\pi / n)} .
$$


From (13) and (14) we see that in order to prove the proposition it is enough to show that

$$
\frac{\sin (\pi / 2(n-1))}{\sin (\pi / n)}<\left(\frac{1}{n}\right)^{\frac{1}{n-1}} \quad \text { for } \quad n \geq 5 .
$$

Numerical checking shows that (15) is true for $n=5,6,7$ or 8 , and so we may assume that $n \geq 9$. Since $0<\frac{\pi}{2(n-1)}<\frac{\pi}{n}<\frac{\pi}{4}$ for $n \geq 5$ and $x \mapsto \frac{\sin x}{\sqrt{x}}$ is an increasing function on $\left(0, \frac{\pi}{4}\right)$ we get that

$$
\frac{\sin (\pi / 2(n-1))}{\sin (\pi / n)}<\sqrt{\frac{n}{2(n-1)}} \quad \text { for } n \geq 5 \text {. }
$$

The sequence $\sqrt{2\left(1-\frac{1}{n}\right)}-n^{\frac{1}{n-1}}$ is clearly increasing for $n \geq 2$, so that

$$
\sqrt{2\left(1-\frac{1}{n}\right)}-n^{\frac{1}{n-1}} \geq \frac{4}{3}-3^{1 / 4}>0
$$

whenever $n \geq 9$. This implies that the right-hand side of (16) is always less than $\left(\frac{1}{n}\right)^{\frac{1}{n-1}}$ if $n \geq 9$, which proves (15).

\section{Second order variations and locally maximal polynomials}

Theorem 3.16 and Lemma 3.17 show that $z^{3}+z$ is a linearly inextensible polynomial which is not a local maximum for $d$ in $S_{n}$. On the other hand, all the properties of the polynomial $p(z)=z^{n}+z$ that we discussed so far seem to suggest that if $n \geq 4$ then $p$ and its rotations could in fact be locally maximal for Sendov's conjecture in the whole class $S_{n}$. More precisely:

(i) $p$ is locally maximal for the function $\left.d\right|_{S(n, 0)}$ (Proposition 2.9);

(ii) $p$ is linearly inextensible (Theorem 3.16);

(iii) $p$ is locally maximal for many types of variations (Proposition 3.20);

(iv) the zeros and critical points of $p$ are symmetrically distributed and satisfy Conjecture 1.3 (Remark 2.6).

We shall now use a second order variational method to show that - contrary to what one might expect from properties (i)-(iv) - the polynomial $p$ is not locally maximal for Sendov's conjecture. Theorems 4.2 and 4.5 below show that if $n=4$ or $n=5$ then $p$ is a kind of inflection point for the function $d$. We conjecture that the same is actually true for all degrees (Remark 4.6) and we also discuss the problem of finding the local maxima for $d$ in $S_{n}$ (Problem 4.8). 
Notation 4.1. Let $a \in[0,1]$ and set (17)

$$
\begin{aligned}
& r=\left|z^{4}+z\right|_{0}=\sqrt[3]{\frac{1}{4}}, \quad \alpha_{1}=\frac{3 \sqrt{3} r}{2-3 r}, \quad \alpha_{2}=-\frac{\sqrt{3}\left[(3 r+2)^{2}+4\right]}{2(3 r-2)^{2}}, \\
& \zeta(a)=\exp \left[i\left(\frac{\pi}{3}+\alpha_{1} a+\alpha_{2} a^{2}\right)\right] .
\end{aligned}
$$

We use the quantities in (17) in order to construct certain continuous deformations of the polynomial $z^{4}+z$. For sufficiently small $a>0$ these perturbations give rise to a one-parameter family of polynomials in $S_{4}$ with the following property:

THeOREm 4.2. Let $\zeta(a)$ be as in (17) and define the polynomial

$$
p_{a}(z):=(z-a)(z+1)[z-\zeta(a)][z-\overline{\zeta(a)}] \in S_{4} .
$$

Then for all sufficiently small $a>0$ one has $\Delta\left(p_{a}, z^{4}+z\right)=\mathscr{O}(a)$ and $d\left(p_{a}\right)=\left|p_{a}\right|_{a}=r+C a^{2}+\mathscr{O}\left(a^{3}\right)$, where $C=\frac{3}{4 r(2-3 r)} \approx 10.81154938$.

In particular, the polynomial $z^{4}+z$ is not locally maximal for Sendov's conjecture.

Proof. Let us first describe the steps we took in order to arrive at the quantities in (17) and the one-parameter family of polynomials defined in (18). We start by introducing six real parameters which we denote by $x_{i}, y_{i}, 1 \leq$ $i \leq 3$, and we define the auxiliary polynomials

$$
\begin{aligned}
& P_{a}\left(z ; x_{1}, x_{2}, x_{3}\right) \\
& \quad=(z-a)\left[z-z_{1}\left(a ; x_{3}\right)\right]\left[z-z_{2}\left(a ; x_{1}, x_{2}\right)\right]\left[z-\overline{z_{2}\left(a ; x_{1}, x_{2}\right)}\right] \\
& \quad=z^{4}+\sum_{j=0}^{3} b_{j}\left(a ; x_{1}, x_{2}, x_{3}\right) z^{j}, \\
& Q_{a}\left(z ; y_{1}, y_{2}, y_{3}\right) \\
& \quad=4\left[z-\omega_{1}\left(a ; y_{3}\right)\right]\left[z-\omega_{2}\left(a ; y_{1}, y_{2}, y_{3}\right)\right]\left[z-\overline{\omega_{2}\left(a ; y_{1}, y_{2}, y_{3}\right)}\right] \\
& \quad=4 z^{3}+\sum_{k=0}^{2} c_{k}\left(a ; y_{1}, y_{2}, y_{3}\right) z^{k},
\end{aligned}
$$


where

(19)

$$
\begin{aligned}
& z_{1}\left(a ; x_{3}\right)=-1+x_{3} a^{2}, \quad z_{2}\left(a ; x_{1}, x_{2}\right)=\exp \left[i\left(\frac{\pi}{3}+x_{1} a+x_{2} a^{2}\right)\right], \\
& \omega_{1}\left(a ; y_{3}\right)=a-\left(r+y_{3} a^{2}\right), \\
& \omega_{2}\left(a ; y_{1}, y_{2}, y_{3}\right)=a+\left(r+y_{3} a^{2}\right) \exp \left[i\left(\frac{\pi}{3}+y_{1} a+y_{2} a^{2}\right)\right] .
\end{aligned}
$$

The idea is now to investigate whether the parameters $x_{i}$ and $y_{i}, 1 \leq i \leq 3$, may be chosen so that the following conditions are satisfied:

(i) $x_{3} \geq 0$ and $y_{3}>0$;

(ii) for all sufficiently small $a>0$ one has

$$
\max _{|z| \leq 1}\left|P_{a}^{\prime}\left(z ; x_{1}, x_{2}, x_{3}\right)-Q_{a}\left(z ; y_{1}, y_{2}, y_{3}\right)\right|=\mathcal{O}\left(a^{3}\right)
$$

where $P_{a}^{\prime}\left(z ; x_{1}, x_{2}, x_{3}\right)$ denotes the derivative of $P_{a}\left(z ; x_{1}, x_{2}, x_{3}\right)$ with respect to $z$. To do this, we expand the coefficients of $P_{a}^{\prime}\left(z ; x_{1}, x_{2}, x_{3}\right)$ and $Q_{a}\left(z ; y_{1}, y_{2}, y_{3}\right)$ into their Maclaurin series so as to get second order approximations of these coefficients (with an error of $\mathscr{O}\left(a^{3}\right)$ ). Let $\tilde{b}_{j}\left(a ; x_{1}, x_{2}, x_{3}\right)$, $1 \leq j \leq 3$, and $\tilde{c}_{k}\left(a ; y_{1}, y_{2}, y_{3}\right), 0 \leq k \leq 2$, be the resulting second degree Maclaurin polynomials in the variable $a$ for the coefficients $b_{j}\left(a ; x_{1}, x_{2}, x_{3}\right)$, $1 \leq j \leq 3$, and $c_{k}\left(a ; y_{1}, y_{2}, y_{3}\right), 0 \leq k \leq 2$, respectively. Then we may write

$$
\begin{aligned}
& (m+1) \tilde{b}_{m+1}\left(a ; x_{1}, x_{2}, x_{3}\right)-\tilde{c}_{m}\left(a ; y_{1}, y_{2}, y_{3}\right) \\
& =\sum_{n=0}^{2} d_{m n}\left(x_{1}, x_{2}, x_{3}, y_{1}, y_{2}, y_{3}\right) a^{n}, \quad 0 \leq m \leq 2,
\end{aligned}
$$

where $d_{m n}\left(x_{1}, x_{2}, x_{3}, y_{1}, y_{2}, y_{3}\right), 0 \leq m, n \leq 2$, are real polynomials in the variables $x_{1}, x_{2}, x_{3}, y_{1}, y_{2}, y_{3}$. Clearly, any solution $\left(x_{1}, x_{2}, x_{3}, y_{1}, y_{2}, y_{3}\right) \in$ $\mathrm{R}^{6}$ to the system of polynomial equations

$$
d_{m n}\left(x_{1}, x_{2}, x_{3}, y_{1}, y_{2}, y_{3}\right)=0, \quad 0 \leq m, n \leq 2,
$$

that satisfies $x_{3} \geq 0$ and $y_{3}>0$ will also satisfy conditions (i) and (ii) above. It turns out that if we let $x_{3}=0$ then system (21) may be reduced to a system of five linear equations in the variables $x_{1}, x_{2}, y_{1}, y_{2}, y_{3}$ which admits a unique solution. We arrive in this way at the following solution to system (21):

$$
\begin{aligned}
& x_{1}=\alpha_{1}, \quad x_{2}=\alpha_{2}, \quad x_{3}=0, \quad y_{1}=\frac{3 \sqrt{3}}{2 r(2-3 r)}, \\
& y_{2}=-\frac{3 \sqrt{3}\left(12 r^{2}+8 r+3\right)}{8 r^{2}(3 r-2)^{2}}, \quad y_{3}=C:=\frac{3}{4 r(2-3 r)},
\end{aligned}
$$


where $r, \alpha_{1}$ and $\alpha_{2}$ are as in (17). Henceforth we assume that the values of the parameters $x_{1}, x_{2}, x_{3}, y_{1}, y_{2}, y_{3}$ are those listed in (22). In particular, this implies that $P_{a}\left(z ; x_{1}, x_{2}, x_{3}\right)$ is the same as the polynomial $p_{a}(z)$ given in (18). Note that by (17) one has $\Delta\left(p_{a}, z^{4}+z\right)=\mathscr{O}(a)$ for all small positive $a$. To simplify the notations, the zeros of the polynomial $Q_{a}\left(z ; y_{1}, y_{2}, y_{3}\right)$ will be denoted by $\omega_{1}(a), \omega_{2}(a)$ and $\overline{\omega_{2}(a)}$, respectively. Then (19) and (22) imply that

$$
\left|\omega_{1}(a)-a\right|=\left|\omega_{2}(a)-a\right|=r+C a^{2} .
$$

It is now practically clear that the desired conclusion should follow from the Newton-Raphson algorithm. We check this in a rigorous way by using the following simple observation.

Lemma 4.3. Let $R$ be a complex polynomial of degree $d \geq 2$. If $w \in \mathrm{C}$ is such that $R^{\prime}(w) \neq 0$ then there exists a zero $z$ of $R$ such that

$$
|w-z| \leq d\left|\frac{R(w)}{R^{\prime}(w)}\right| .
$$

Proof. If $R(w)=0$ there is nothing to prove. Otherwise, we let $z_{1}, \ldots, z_{d}$ denote the zeros of $R$. Then

$$
\sum_{i=1}^{d} \frac{1}{\left|w-z_{i}\right|} \geq\left|\sum_{i=1}^{d} \frac{1}{w-z_{i}}\right|=\left|\frac{R^{\prime}(w)}{R(w)}\right|,
$$

which proves the lemma.

Let $w_{1}(a), w_{2}(a)$ and $w_{3}(a)$ denote the critical points of the polynomial $p_{a}(z)$. Since $p_{a}(z) \rightarrow z^{4}+z$ as $a \rightarrow 0$, we may label these critical points so that $w_{1}(a) \in \mathrm{R}, \Im\left(w_{2}(a)\right)>0$ and $w_{3}(a)=\overline{w_{2}(a)}$ if $a$ is positive and sufficiently small. A straightforward computation shows that

$$
p_{a}^{\prime \prime}\left(\omega_{1}(a)\right)=\frac{3}{r}+\mathscr{O}(a) \quad \text { and } \quad p_{a}^{\prime \prime}\left(\omega_{2}(a)\right)=\overline{p_{a}^{\prime \prime}\left(\overline{\omega_{2}(a)}\right)}=\frac{3 e^{\frac{2 \pi i}{3}}}{r}+\mathscr{O}(a)
$$

which combined with Lemma 4.3 and condition (ii) implies that

$$
w_{1}(a)=\omega_{1}(a)+\mathscr{O}\left(a^{3}\right) \quad \text { and } \quad w_{2}(a)=\overline{w_{3}(a)}=\omega_{2}(a)+\mathscr{O}\left(a^{3}\right) .
$$

The desired result is now a consequence of (23).

As one may expect, the second order variational method that we used in the proof of Theorem 4.2 works even in more general cases. However, for higher 
degrees the procedure described above requires an increasingly large amount of computations. Tedious as they may be when done by hand, for small degrees these computations become relatively easy if one uses for instance a Maple computer program. Such a program has considerably simplified our task in the course of proving Theorem 4.2 and it also allowed us to obtain a similar result for the polynomial $z^{5}+z$.

Notation 4.4. Let $a \in[0,1]$ and set

$$
\begin{aligned}
& s=\left|z^{5}+z\right|_{0}=\sqrt[4]{\frac{1}{5}}, \quad \beta=\frac{2 \sqrt{2} s^{2}}{1-2 s^{2}}, \quad \gamma=\frac{4 \sqrt{2}}{5 s\left(1-2 s^{2}\right)}, \\
& \delta=\frac{60 s^{4}-19}{50 s^{2}\left(2 s^{2}-1\right)^{2}}, \quad K=\frac{2}{5 s\left(1-2 s^{2}\right)} \approx 5.665658792, \\
& \eta(a)=\exp \left[i\left(\frac{\pi}{4}+\beta a\right)\right], \\
& \chi_{1}(a)=a+\left(s+K a^{2}\right) \exp \left[i\left(\frac{\pi}{4}+\gamma a+\delta a^{2}\right)\right], \\
& \chi_{2}(a)=a+\left(s+K a^{2}\right) \exp \left[i\left(\frac{3 \pi}{4}+\gamma a-\delta a^{2}\right)\right] .
\end{aligned}
$$

We use these quantities in order to define the following one-parameter families of polynomials:

$$
\begin{aligned}
& q_{a}(z):=(z-a)(z-\eta(a))(z-i \eta(a))[z-\overline{\eta(a)}][z+i \overline{\eta(a)}] \in S_{5}, \\
& s_{a}(z):=5\left(z-\chi_{1}(a)\right)\left(z-\chi_{2}(a)\right)\left[z-\overline{\chi_{1}(a)}\right]\left[z-\overline{\chi_{2}(a)}\right] .
\end{aligned}
$$

We can now formulate an analogue of Theorem 4.2 for the polynomial $z^{5}+z$. Its proof is similar to that of Theorem 4.2 and is therefore omitted.

THEOREM 4.5. For all sufficiently small $a>0$ one has

$$
\Delta\left(q_{a}, z^{5}+z\right)=\mathscr{O}(a) \quad \text { and } \quad \Delta\left(\frac{q_{a}^{\prime}}{5}, \frac{s_{a}}{5}\right)=\mathscr{O}\left(a^{3}\right) \text {, }
$$

so that $d\left(q_{a}\right)=\left|q_{a}\right|_{a}=s+K a^{2}+\mathcal{O}\left(a^{3}\right)$. In particular, the polynomial $z^{5}+z$ is not locally maximal for Sendov's conjecture.

REMARK 4.6. In view of Lemma 3.17 and Theorems 4.2-4.5 it seems reasonable to conjecture that polynomials of the form $z^{n}+z$ or, more generally, the 0 -maximal polynomials of degree $n \geq 2$ given in Theorem 2.4 are not local maxima for the function $d$. One could for instance try to find an algorithmic 
proof of this conjecture based on the second order variational method that we just described.

REMARK 4.7. Theorems $4.2-4.5$ and the second order variational methods used in [9], [10] and [20] clearly expose the limitations of what can be achieved through first order expansions and show the necessity of using higher order approximations when dealing with problems such as Sendov's conjecture.

The polynomials in Conjecture 1.2 - i.e., the conjectured extremal polynomials for Sendov's conjecture - have long been the only known examples of local maxima for $d$ in $S_{n}$ (cf. [9] and [20]). Moreover, Sendov's conjecture and related problems have been studied almost exclusively by means of analytical or variational methods and all the results obtained until very recently seemed to suggest a negative answer to the following question:

Problem 4.8. Are there any local maxima for $d$ in $S_{n}$ other than $z^{n}+e^{i \theta}$, $\theta \in \mathbf{R}$ ?

As pointed out in [2], the fact that $d$ fails to be a (logarithmically) plurisubharmonic function in the polydisk $\bar{D}^{n}$ accounts for many of the difficulties in answering Problem 4.8. Quite recently Miller showed in [11] that the answer to this question is in fact affirmative by explicitly constructing local maxima of degree $n \in\{8,12,14,20,26\}$ which are "unexpected" in the sense that they are not of the form $z^{n}+a$ with $|a|=1$. In particular, Miller's result shows that local variational methods alone (of any order) are not enough for dealing with Conjecture 1.2 in full generality. It is therefore important to consider alternative methods for studying Sendov's conjecture and its strong version (Conjecture 1.2). An inductive approach to Conjectures 1.1 and 1.2 based on apolarity and coincidence theorems of Grace-Walsh type was discussed in [2]. More recently, operator theoretical interpretations and extensions of Sendov's conjecture and related questions were proposed in [3], [4], [5]. In loc. cit. it was also shown that Conjectures 1.1 and 1.2 may in fact be viewed as part of the more general problem of describing the relationships between the spectra of normal matrices and the spectra of their degeneracy one principal submatrices. These and similar questions will be addressed in a series of forthcoming papers.

\section{REFERENCES}

1. Bojanov, B., Rahman, Q. I., Szynal, J., On a conjecture about the critical points of a polynomial, in Delay Equations, Approximation and Application (G. Meinardus, G. Nürnberger, Eds.), 83-93, Internat. Ser. Numer. Math. 74 (1985).

2. Borcea, J., Two approaches to Sendov's conjecture, Arch. Math. 71 (1998), 46-54.

3. Borcea, J., Maximal and inextensible polynomials and the geometry of the spectra of normal operators, arXiv:math.CV/0309233. 
4. Borcea, J., Equilibrium points of logarithmic potentials induced by positive charge distributions. I. Generalized de Bruijn-Springer relations, Trans. Amer. Math. Soc., to appear; arXiv:math.CV/0601519.

5. Borcea, J., Equilibrium points of logarithmic potentials induced by positive charge distributions. II. A conjectural Hausdorff geometric symphony, submitted; preprint (2005).

6. Brown, J. E, Xiang, G., Proof of the Sendov conjecture for polynomials of degree at most eight, J. Math. Anal. Appl. 232 (1999), 272-292.

7. Miller, M. J., Maximal polynomials and the Ilieff-Sendov conjecture, Trans. Amer. Math. Soc. 321 (1990), 285-303.

8. Miller, M. J., Continuous independence and the Ilieff-Sendov conjecture, Proc. Amer. Math. Soc. 115 (1992), 79-83.

9. Miller, M. J., On Sendov's conjecture for roots near the unit circle, J. Math. Anal. Appl. 175 (1993), 632-629.

10. Miller, M. J., A quadratic approximation to the Sendov radius near the unit circle, Trans. Amer. Math. Soc. 357 (2005), 851-873.

11. Miller, M. J., Unexpected local extrema for the Sendov conjecture, arXiv:math.CV/0505424.

12. Phelps, D., Rodriguez, R. S., Some properties of extremal polynomials for the Ilieff conjecture, Kôdai Math. Sem. Rep. 24 (1972), 172-174.

13. Rahman, Q. I., Schmeisser, G., Analytic Theory of Polynomials, London Math. Soc. Monogr. (N. S.) 26 (2002).

14. Rockafellar, R. T., Convex Analysis, Princeton Univ. Press, Princeton, NJ, 1997.

15. Rubinstein, Z., On a problem of Ilyeff, Pacific J. Math. 26 (1968), 159-161.

16. Schmeisser, G., The conjectures of Sendov and Smale, in Approximation Theory: A volume dedicated to Blagovest Sendov (B. Bojanov, Ed.), 353-369, Darba, Sofia, 2002.

17. Schmieder, G., Szynal, J., On the distribution of the derivative zeros of a complex polynomial, Complex Variables Theory Appl. 47 (2002), 239-241.

18. Sendov, B., Hausdorff geometry of polynomials, East J. Approx. 7 (2001), 123-178.

19. Sheil-Small, T., Complex Polynomials, Cambridge Stud. Adv. Math. 75 (2002).

20. Vâjâitu, V., Zaharescu, A., Ilyeff's conjecture on a corona, Bull. London Math. Soc. 25 (1993), 49-54.

DEPARTMENT OF MATHEMATICS

STOCKHOLM UNIVERSITY

SE-106 91 STOCKHOLM

SWEDEN

E-mail: julius@math.su.se 